\title{
A Near-IR Survey of the Southern Galactic Plane
}

\author{
D. Jack MacConnell
}

Astronomy Programs, Computer Sciences Corporation, Space Telescope Science Institute

\author{
Robert F. Wing \\ Ohio State University \\ Edgardo Costa H. \\ Universidad de Chile
}

\begin{abstract}
We describe a long term project to find faint, heavily-reddened, cool supergiants and carbon stars and to classify IRAS Point Sources within $6^{\circ}$ of the southern galactic plane. Many new supergiants and carbon stars have been found, and thousands of IRAS Point Sources have had their spectral types determined.
\end{abstract}

\section{The Plate Material}

\begin{abstract}
About 450 plates were taken by DJM for a near-infrared survey of the southern Milky Way using the $61 / 91 \mathrm{~cm}$ Curtis Schmidt camera of The University of Michigan located at Cerro Tololo Interamerican Observatory. The emulsion used was Kodak I - N behind an RG 680 filter giving a spectral coverage of $6800 \AA$ to $8800 \AA$. The $6^{\circ}$ and $4^{\circ}$ prisms were mounted with their apices rotated $180^{\circ}$ to give the dispersion of a $2^{\circ}$ prism; this combination produces spectra $0.5 \mathrm{~mm}$ long with a dispersion about $3400 \AA / \mathrm{mm}$ at the telluric $\mathrm{A}$ - band. Despite the small plate scale of $96^{\prime \prime} 6 / \mathrm{mm}$, spectrum overlaps are not a problem at this low dispersion. Between 1969 and 1971, unwidened exposures of 30 and 5 minutes were made on separate plates centered every $4^{\circ}$ in longitude at $b=0^{\circ}$ and at $b= \pm 4^{\circ}$ for a total of 138 fields. Starting in 1972, 60-min, ammonia-sensitized plates were taken on all of the $b=0^{\circ}$ centers and on many off the plane. The approximate limiting I magnitudes are 9,11 , and 13 for the $5-, 30-$, and $60-\mathrm{min}$ exposures, respectively.
\end{abstract}

\section{Search for Red Supergiants}

The original objective of the survey was to extend the near-infrared surveys of Nassau, Blanco, \& Morgan (1954) and Blanco \& Münch (1955) further south and to fainter limiting mags. They identified heavily-reddened, early $M$ stars on their plates which show a characteristic tapered spectrum with the point toward shorter wavelengths and suggested that many of these might be $M$ supergiants. 
Slit spectra taken by others confirmed their suggestion. Such stars are important for visual mapping of galactic structure, and their numbers compared with those of blue supergiants and WR stars can be used to trace the chemical enrichment history of the Galaxy (Meylan \& Maeder 1983). Red supergiants have their peak energy distribution in the near-infrared, and the interstellar absorption is only $1 / 3$ of its value in the blue, so reddened, cool supergiants may be seen up to several kiloparsecs. A thorough discussion of our program appeared in MacConnell et al. (1992), and further papers in the series will be published in the Astronomical Journal .

Plates are scanned visually by DJM using a binocular microscope at a magnification of 12 diameters. Each plate is examined independently of others on that center searching for stars having a tapered form and also for carbon stars. Stars with strong TiO bands are expected to be normal $\mathrm{M}$ giants and are not marked. Stars noted as of interest on separate plates of the same field are reexamined on the best deep plate, and a single list of candidates is compiled for that field.

We have been gathering observations of supergiant candidates for nearly 10 years using the facilities of Cerro Tololo, La Silla, and Las Campanas Observatories. These follow-up data are of two types:

- 8-color photometry on the near-infrared, narrow-band system of Wing (1971) which permits good temperature and luminosity classification for stars cooler than $\mathrm{K} 3.5$; the system determines temperature type from the strength of TiO at $7054 \AA$ and luminosity from the $(3,1)$ and $(0,0) \mathrm{CN}$ bands at $8120 \AA$ and $10975 \AA$ respectively, and is carefully calibrated to the MK system. We have photometric measures of over 900 candidates; Wing is responsible for the reductions.

- CCD spectra at $4 \AA /$ pixel have been taken of over 500 candidate stars together with spectra of several dozen equatorial and southern MK standards. The spectra cover the range $6400 \AA$ to $8800 \AA$ and are used to eliminate $\mathrm{S}$ stars (presence of $\mathrm{ZrO}$ band at $6474 \AA$ ), to serve as an independent check on the photometric classification, and for classification of candidates earlier than K3. The lower panel of Fig. 1 shows the observed spectrum of the reddened program star X -46 and the continuum fitted to it using tasks in the IRAF image-processing program. The upper panel shows the result after dividing by the continuum, and the flattened spectrum may then be compared with similar spectra of standard stars; this star is classified as M0.1 Ib by the photometry and about M1.0 Ib from the spectrum.

Plate-scanning, follow-up observations, and reductions are in progress, and we will soon publish a paper on our results in the sector $208^{\circ} \leq l^{I} \leq 248^{\circ}$. We have found about 120 new $\mathrm{K}$ and $\mathrm{M}$ supergiants to $\mathrm{V} \sim 15$ thus more than doubling the number known in that region.

\section{Classification of IRAS Point Sources}

The same plates have also been used to classify IRAS Point Sources (PS) along the southern galactic plane. DJM has classified over 14,000 PS having 


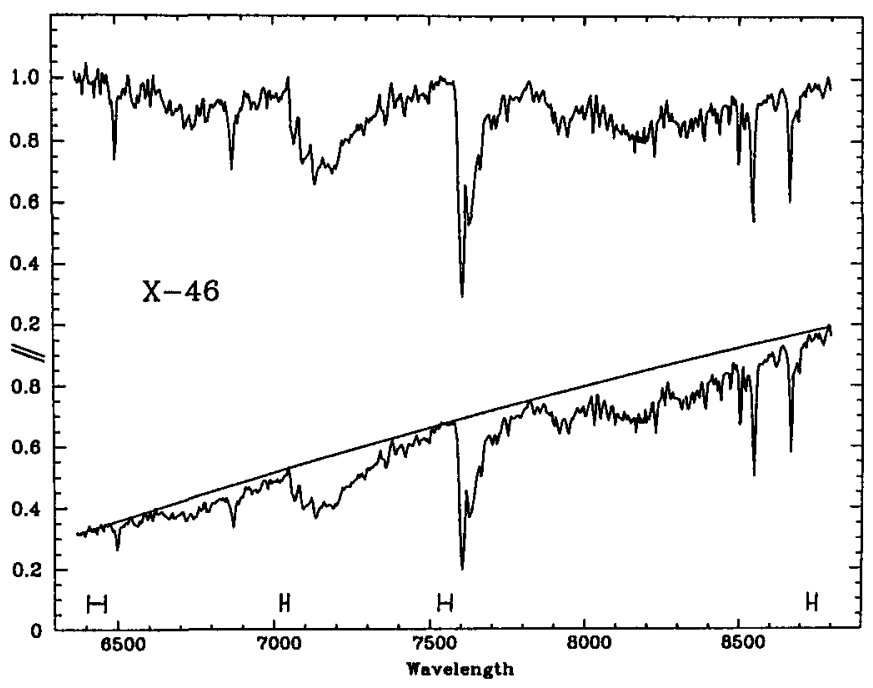

Figure 1. The lower panel shows the observed spectrum of a reddened program star with the continuum fitted to it using tools in IRAF. The upper panel presents the flattened spectrum of this star after dividing by the fitted continuum. It is classified as M1 Ib by comparison with standard star spectra.

$\mathrm{F}(12 \mu \mathrm{m}) \geq \mathrm{F}(25 \mu \mathrm{m})$ using a set of overlays to the Schmidt plate scale and centers; we use a grid of SAO stars to register the plate on the overlay - see Fig. 2 for a reproduction of the plate and overlay for $l^{I t}=286^{\circ}, b^{I I}=0^{\circ}$. The IRAS PS positional uncertainty ellipse for a $12 \mu \mathrm{m}$ source is typically $2 \prime 5 \times 12^{\prime \prime}$, and a late-type star is usually found within that area. Most of these stars are not in catalogues, so there is no previous association with IRAS Point Sources. The stars may be classified into about 9 categories including four divisions among the $M$ stars; the majority of the objects are normal-appearing AGB stars of the $\mathrm{O}$ - and $\mathrm{C}$-rich sequences. The low resolution of the spectra does not permit luminosity classification, but most of the PS classified are undoubtedly giants. We have found some cases where a PS has been associated in the PS Catalogue with a bright, early-type star some tens of arcsecs away whereas the correct identification is with a faint cool star at the exact IRAS position. There are also cases where the PS position falls between two cool stars, and, in about 9 per cent of the cases, there is no image seen at the PS position.

DJM would be pleased to make a complete or partial listing of the PS classified available to anyone interested. 


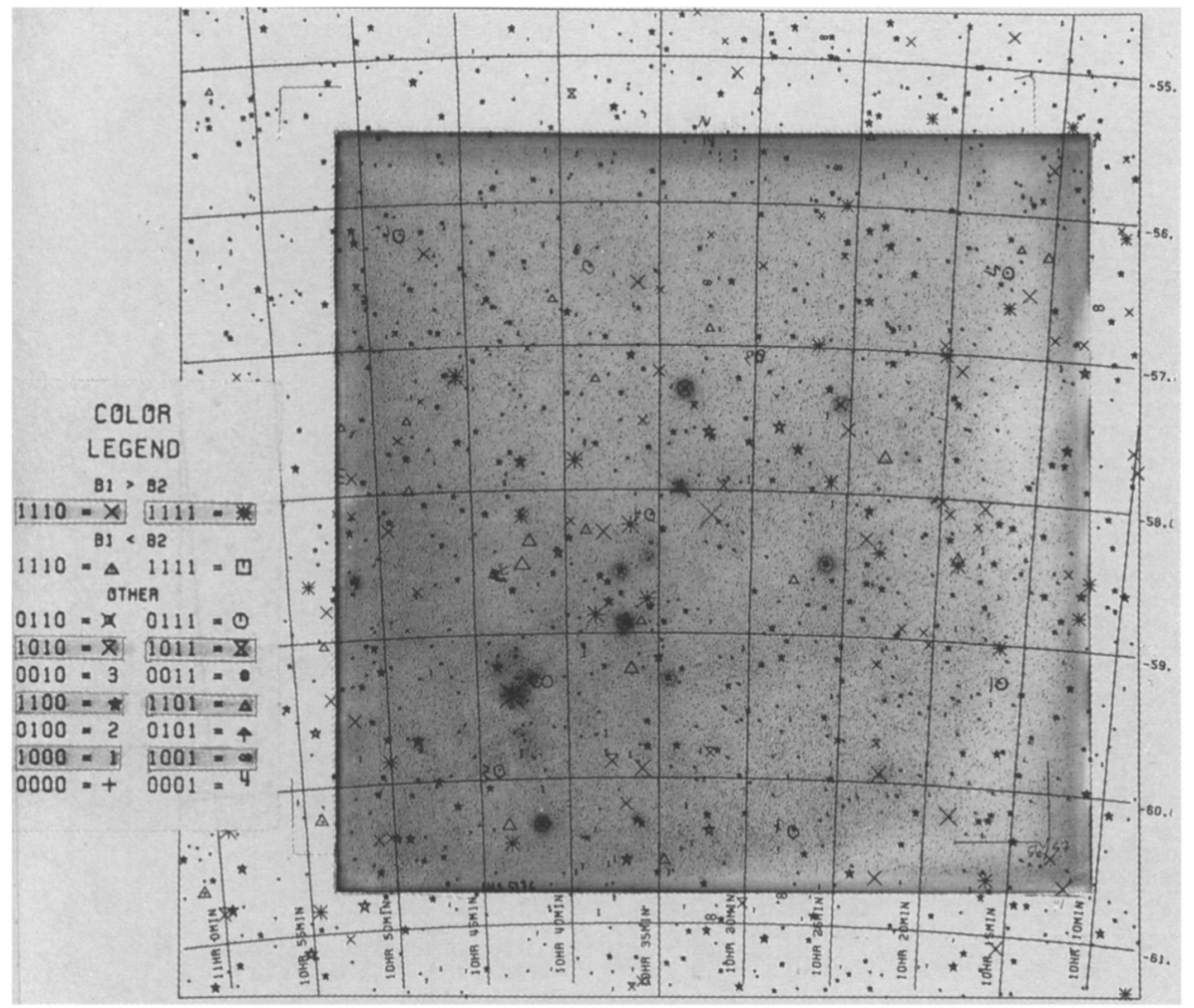

Figure 2. This figure shows an objective-prism plate superposed on an overlay to the same scale of the IRAS Point Sources in the field having $\mathrm{F}(12 \mu \mathrm{m}) \geq \mathrm{F}(25 \mu \mathrm{m})$. The plate covers $5: 1$ on a side. Symbols are explained on the Color Legend, and those pertinent to this program have been highlighted.

\section{References}

Blanco V. M. \& Münch L., 1955, Bol. Ton. Tac., No. 12, 17

MacConnell D. J., Wing R. F. \& Costa E., 1992, AJ, 104, 821

Meylan G. \& Maeder A., 1983, A\&A, 124, 84

Nassau J. J., Blanco V. M. \& Morgan W. W., 1954, ApJ, 120, 478

Wing R. F., 1971, in Proceedings of the Conference on Late-Type Stars, G. W. Lockwood \& H. M. Dyck, eds, KPNO Contrib. No. 554, 145 